\title{
ON IDEALS OF SETS AND THE POWER SET OPERATION
}

\author{
BY THOMAS JECH ${ }^{1}$ AND KAREL PRIKRY ${ }^{2}$
}

\author{
Communicated by S. Feferman, February 16, 1976
}

We present some inequalities involving cardinal powers. In most of the results we assume the existence of an ideal $I$ satisfying a weak completeness condition.

For the remainder of this paper, $I$ will always denote an ideal over $\omega_{1}$ containing all enumerable sets. $F \subseteq P\left(\omega_{1}\right)$ is $I$-disjoint if $X \cap Y \in I$ for all distinct $X, Y \in F ; F$ is almost disjoint if $|X \cap Y| \leqslant \aleph_{0}$ for all distinct $X, Y \in F . I$ is $\lambda$-saturated if $|F|<\lambda$ for every $I$-disjoint $F \subseteq P\left(\omega_{1}\right)-I$.

THEOREM 1. Let I be $\sigma$-additive. If $2^{\aleph_{0}}<2^{\aleph_{1}}$ and $2^{\aleph_{0}}<\aleph_{\omega_{1}}$, then for every $\lambda<2^{\aleph_{1}}$ there is an almost disjoint $F \subseteq P\left(\omega_{1}\right)-I$ with $|F|=\lambda$. Moreover, if $2^{\aleph_{1}}$ is singular, we get such an $F$ with $|\bar{F}|=2^{\aleph_{1}}$. Hence if $2^{\aleph_{0}}<2^{\aleph_{1}}$ and $2^{\aleph_{0}}<\aleph_{\omega_{1}}$, then there exists no $\lambda$-saturated ideal for any $\lambda<2^{\aleph_{1}}$.

REMARK. In [1] the same assumption on $2^{\aleph 0}$ is used to obtain an almost disjoint $F$ such that $|F|=2^{\aleph_{1}}$. In [3] stronger assumptions on $2^{\aleph_{0}}$ are used to show that the ideal of nonstationary sets is not $\aleph_{2}$-saturated.

For $S \in P\left(\omega_{1}\right)-I, W$ is an I-partition of $S$ if $W$ is a maximal $I$-disjoint family $\subseteq P(S)-I$. If $W_{0}$ and $W_{1}$ are $I$-partitions of $S$, then $W_{1}$ is a refinement of $w_{0}$ if every $X \in W_{1}$ is included in some $Y \in W_{0}$.

$I$ is precipitous if for every $S \in P\left(\omega_{1}\right)-I$, and every sequence $\omega_{n}(n \in \omega)$ of $I$-partitions of $S$ such that $W_{n+1}$ is a refinement of $W_{n}$, there exists a sequence $X_{n} \in W_{n}$ such that $X_{n+1} \subseteq X_{n}$ and $\bigcap\left\{X_{n}: n \in \omega\right\} \neq 0$.

Proposition. If there is a precipitous $I$, then there is a $\sigma$-additive, normal, precipitous I. If I is normal and precipitous, then $\omega_{1}$ is measurable in $L[I]$. If $I$ is $\aleph_{2}$-saturated, then $I$ is precipitous. The ideal $\left\{X \subseteq \omega_{1}:|X| \leqslant \aleph_{0}\right\}$ is not precipitous.

We shall consider a class of cardinal functions called nice functions. The following functions are nice: $\Phi(\alpha)=\omega_{\alpha} ; \Phi(\alpha)=$ the $\alpha$ th weakly inaccessible cardinal. If $\Phi$ and $\psi$ are nice, then so are, for example, $\psi_{1}(\alpha)=$ the $\alpha$ th fixed point of $\Phi ; \psi_{2}(\alpha)=\Phi(\alpha+\alpha) ; \psi_{3}(\alpha)=\Phi(\psi(\alpha))$.

\footnotetext{
AMS (MOS) subject classifications (1970). Primary $02 \mathrm{~K} 35$.

${ }^{1}$ Research supported by NSF Grant MPS75-07408.

${ }^{2}$ Research supported by NSF Grant GP -43841 .
}

Copyright $\odot$ 1976, American Mathematical Society 
THEOREM 2. Suppose that there is a precipitous ideal I in $P\left(\omega_{1}\right)$. Let $\Phi$ be a nice cardinal function and let $\kappa$ be a strong limit cardinal such that $\operatorname{cf}(\kappa)=\omega_{1}$.

(a) If $\kappa=\Phi\left(\omega_{1}\right)$, then $2^{\kappa}<\Phi\left(\left(2^{\aleph_{1}}\right)^{+}\right)$.

(b) If $\sup \{\Phi(\alpha): \Phi(\alpha)<\kappa\}<\kappa$, then $2^{\kappa} \leqslant \min \{\Phi(\alpha)$ : $\Phi(\alpha)>\kappa\}$.

Suppose that $I$ is also $\lambda$-saturated. Then

(c) If $\kappa=\Phi\left(\omega_{1}\right)$, then $2^{\kappa}<\Phi(\lambda)$.

(d) If $2^{\aleph_{0}} \leqslant \Phi\left(\omega_{1}\right)$ and $\lambda<\Phi(\lambda)$, then $2^{\aleph_{1}} \leqslant \Phi(\lambda)$.

(e) If $2^{\aleph_{0}} \leqslant \Phi\left(\omega_{1}\right)$ and $\lambda=\Phi(\lambda)$, then $2^{\aleph} 1 \leqslant \Phi(\lambda+\lambda)$.

(f) If $2^{\aleph_{0}}<\Phi(\alpha)$, where $\alpha<\omega_{1}$, then $2^{\aleph_{1}}$ is less than the $\alpha$ th value of $\Phi$ above $\lambda$.

For instance, if $\Phi$ is the enumeration of fixed points of the aleph-function, (a) and (c) give estimates for a case left open in [2].

The assumption of niceness of $\Phi$ is necessary. Using the forcing constructions of [5], [6] and of Silver we can show: If $\kappa$ is a super-compact cardinal and $\nu>\kappa$ arbitrary, then there exists a generic extension which preserves cardinals, and in which $\kappa$ is a strong limit cardinal of cofinality $\omega_{1}$, and $2^{\kappa}>\nu$.

We have two methods to prove our results. The first method uses a generic ultrapower-a combination of forcing and ultrapowers. This method is related to Silver's work on the singular cardinal problem [7] and Solovay's and Kunen's work on saturated ideals [8], [4].

We work in a given model $M$. Let $I$ be a $\sigma$-additive ideal over $\omega_{1}$ in $M$. We consider a generic extension corresponding to the partial ordering $\left\langle P\left(\omega_{1}\right) / I, \subseteq\right\rangle$. A generic set $G$ is an ultrafilter (w.r.t. $M$ ). In $M[G]$ we consider the ultrapower $N=\operatorname{Ult}(M, G)$ and obtain an elementary embedding $i: M \rightarrow N$. We use the lemma that $N$ is well founded iff $I$ is precipitous.

The second method is elementary in the spirit of [2] and uses almost disjoint transversals (a.d.t). Let $I$ be $\sigma$-additive. An $I$-function is an ordinal function $f$ such that $\operatorname{dom}(f) \in P\left(\omega_{1}\right)-I$. We set $f<g$ if $\operatorname{dom}(f) \subseteq \operatorname{dom}(g)$ and $f(\alpha)<$ $g(\alpha)$ for all $\alpha \in \operatorname{dom}(f)$.

An $I$-function $f$ has $\kappa$ almost disjoint transversals if there exists a family $F$ of $I$-functions such that: $|F| \geqslant \kappa ; g<f$ for all $g \in F$; and if $g, h \in F$ and $g \neq h$, then $\{\alpha: g(\alpha)=h(\alpha)\} \in I$.

We make repeated use of the following lemmas.

Lemma 1. Suppose that $f$ has $\kappa$ a.d.t. and $I$ is $\lambda$-saturated.

(a) If $\kappa \geqslant \lambda$, then for every $\nu<\kappa$ there exists $g<f$ which has $\nu$ a.d.t.

(b) If $\mathrm{cf}(\kappa) \geqslant \lambda$, then there exists $g \subseteq f$ such that for all $h \subseteq g$, $h$ has к a.d.t.

LEMMA 2. If I is precipitous and $F$ is a nonempty family of I-functions 
closed under restrictions then there exists $f \in F$ such that there is no $g \in F$ with $g<f$.

REMARK. After we had announced the above results, W. Mitchell proved that if it is consistent that a measurable cardinal exists, then it is consistent that $\omega_{1}$ carries a precipitous ideal. In view of this result and of the Proposition above, it appears that the existence of a precipitous ideal over $\omega_{1}$ is the exact counterpart of measurability, suitable for $\omega_{1}$. This seems to give added interest to the results presented above.

\section{REFERENCES}

1. J. E. Baumgartner, Ph.D. Thesis, University of Calif., Berkeley, 1970.

2. F. Galvin and A. Hajnal, Inequalities for cardinal powers, Ann. of Math. (2) 101 (1975), 491-498.

3. J. Ketonen, Some combinatorial principles, Trans. Amer. Math. Soc. 188 (1974), 387-394. MR 48 \#10808.

4. K. Kunen, Some applications of iterated ultrapowers in set theory, Ann. Math. Logic 1 (1970), 179-227. MR 43 \#3080.

5. M. Magidor, Changing cofinalities of cardinals (to appear).

6. T. Menas, Ph.D. Thesis, Univ. of Calif., Berkeley, 1973.

7. J. Silver, On the singular cardinals problem, Proc. Internat. Congr. Mathematicians, (Vancouver, 1974), vol. 1, 265-268.

8. R. M. Solovay, Real-valued measurable cardinals, Axiomatic Set Theory (Proc. Sympos. Pure Math., vol. 13, Part 1), Amer. Math. Soc., Providence, R. I., 1971, pp. 397428. MR $45 \# 55$.

DEPARTMENT OF MATHEMATICS, PENNSYLVANIA STATE UNIVERSITY, UNIVERSITY PARK, PENNSYLVANIA 16802

SCHOOL OF MATHEMATICS, UNIVERSITY OF MINNESOTA, MINNEAPOLIS, MINNESOTA $\mathbf{5 5 4 5 5}$ 